\title{
Calculating Absorption Shifts for Retinal Proteins: Computational Challenges
}

\section{- Supporting Information -}

\author{
M. Wanko, ${ }^{1}$ M. Hoffmann, ${ }^{1}$ P. Strodel $,{ }^{1}, 2$ W. Thiel, ${ }^{3}$ F. Neese, ${ }^{4}$ T. Frauenheim,${ }^{1}$ and M. Elstner ${ }^{1,2, *}$ \\ ${ }^{1}$ Dept. of Theoretical Physics, University of Paderborn, D-33098 Paderborn, Germany \\ ${ }^{2}$ German Cancer Research Center, Dept. Molecular Biophysics, D-60120 Heidelberg, Germany \\ ${ }^{3}$ MPI für Kohlenforschung, D-45470 Mühlheim an der Ruhr, Germany \\ ${ }^{4}$ MPI für Bioanorganische Chemie D-45470 Mühlheim an der Ruhr, Germany
}

\section{CALIBRATION OF THRESHOLDS WITHIN THE SORCI METHOD}

For all thresholds used within the SORCI method appropriate values were determined in order to achieve a maximum of accuracy in the excitation energies of the lowest two valence states of the PSB of retinal and its model systems. The $S_{1}$ energy was requested to be converged within $0.1 \mathrm{eV}$ of the method's limitation for a five conjugated double bond model in vacuum as well as in the polarizing field of a counter ion. For this benchmark we used the structure published in Ref. 1, including the chromophore as PSB5 model and point charges of the principal counter ion (see Fig. 1). For further information about thresholds within the SORCI method see Ref. 2.

The range of active (unfrozen) orbitals that enter the CI calculation can be set via an energy criterion $\left(E_{w i n}\right)$. For occupied orbitals a lower threshold of 3 hartree was chosen which freezes only the core orbitals. An upper threshold limits the number of virtual orbitals. The latter is not computationally critical since the number of approximated natural orbitals that enter the final CI calculation is limited by the threshold $T_{\text {nat }}$ (see below). However, in order to reduce the memory required for integral storage, a threshold of 3 hartree can be used without loose of accuracy in case of PSB5 (see Table 2). This value can also be used safely for the complete chromophore which has a lower ionization potential than the truncated models.

The reference space has been constituted by all configurations from a RAS ( 6 electrons in $6 \pi$ orbitals with up to triple excitations) that contribute to any of the requested three roots with a coefficient larger than $T_{\text {Prediag. }}$ Fig. 3 shows that SORCI excitation energies for this system are not very sensitive to the size of the reference space. Surely, the accuracy could be improved by using huge reference spaces, but this is less efficient than to improve e.g. $T_{\text {Sel }}$. The strategy of SORCI is to use a small reference space and take care of strong perturbing configurations in an additional variational step. The least CPU time was not used with

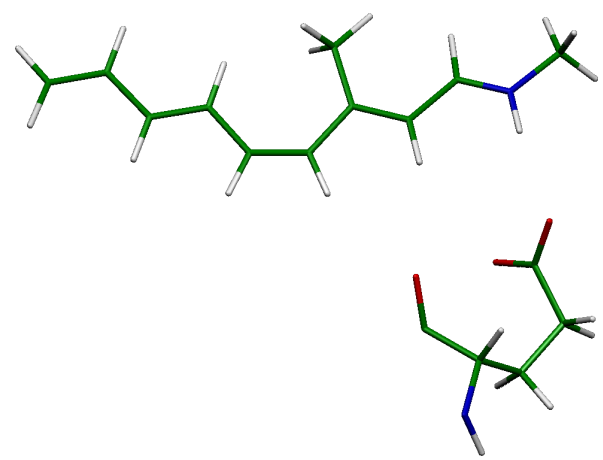

FIG. 1: The setup of Ref. 1 used for calibration of SORCI thresholds; the Glu113 residue is represented by point charges. 


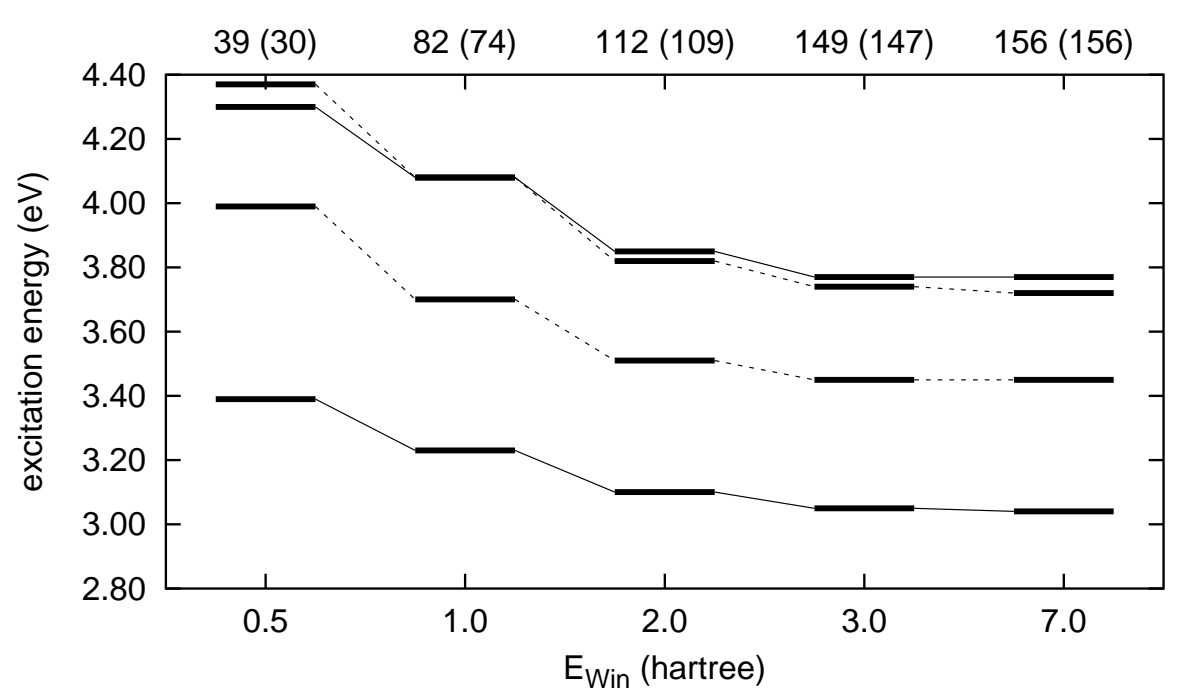

FIG. 2: Dependence of vertical excitation energies $(\mathrm{eV})$ on the range of non-frozen orbitals via energy threshold $E_{\text {Win. }}$ Upper line: number of non-frozen virtual orbitals for PSB5 and PSB5+Glu133 (in parentheses). Levels of the system PSB5+Glu133 are connected via dashed lines.

the smallest number of configurations, but with $T_{\text {Prediag }}=10^{-3}$ which we used for all calculations in this work.

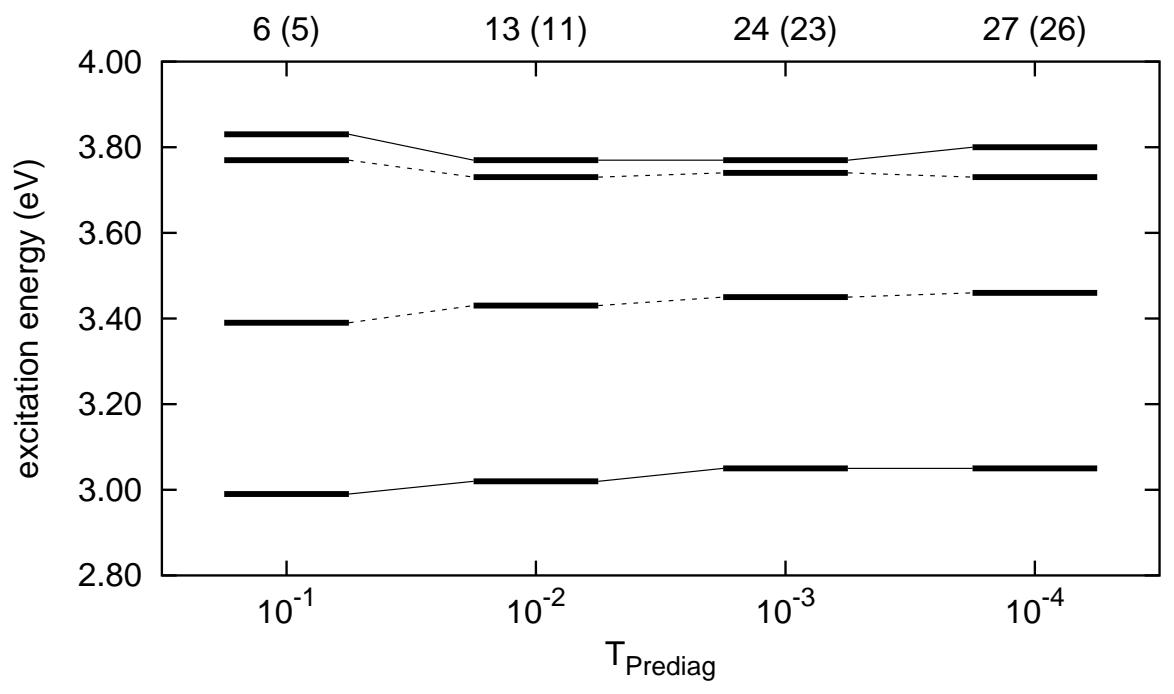

FIG. 3: Dependence of vertical excitation energies $(\mathrm{eV})$ on the reference space selection threshold $T_{\text {Prediag. }}$ Upper line: number of configurations in the reference space (final MRDDCI3 calculation), values for PSB5 and PSB5+Glu133 (in parentheses). Levels of the system PSB5+Glu133 are connected via dashed lines.

Approximated natural orbitals are kept frozen in the final calculation when their occupation numbers are below the threshold $T_{\text {Nat }}$. As Fig. 4 shows, convergency is achieved between values of $10^{-5}$ and $10^{-6}$. The latter has been chosen for all calculations in this work.

The selection threshold $T_{\text {Sel }}$ which divides the first-order interacting space into subspaces for the variational und second-order perturbational treatment is critically with respect to CPU time. As Fig. 5 indicates, excitation energies are not entirely converged at $T_{\text {Sel }}=10^{-6}$. Though we have chosen this value for our cal- 


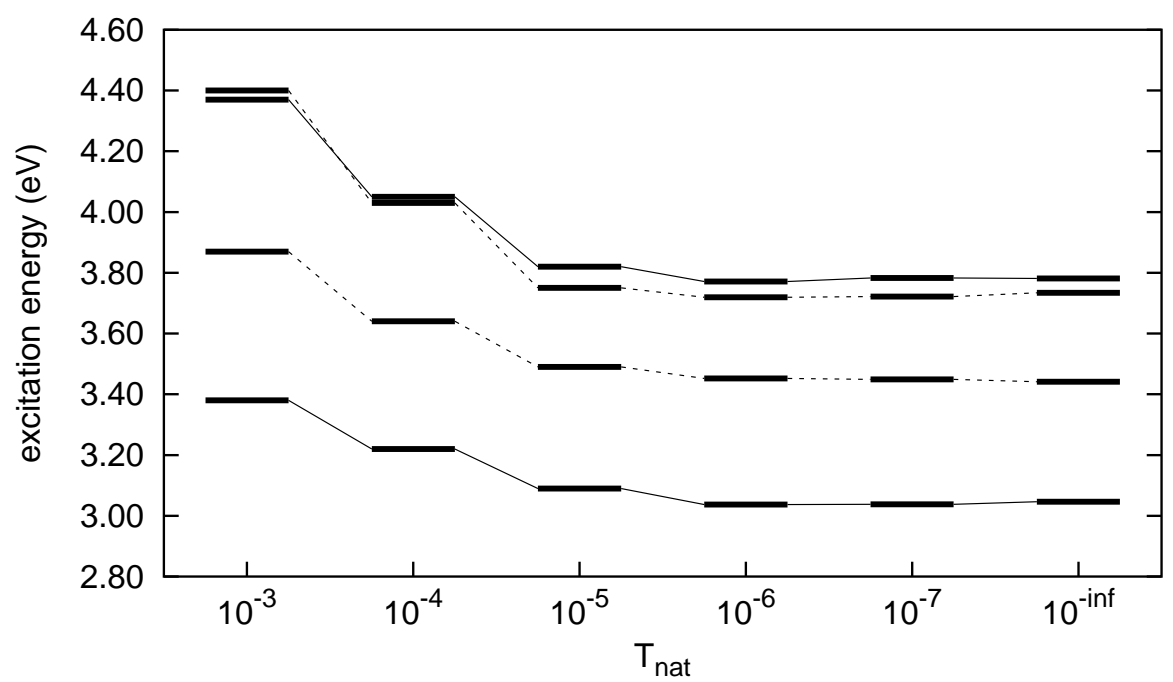

FIG. 4: Dependence of vertical excitation energies $(\mathrm{eV})$ on the natural orbital threshold $T_{\mathrm{Nat}}$. Levels of the system PSB5+Glu133 are connected via dashed lines.

culations as an appropriate compromise between accuracy and efficiency for two reasons: First, the increase of the $S_{1}$ excitation energy with tightening of the selection threshold (beyond $10^{-6}$ ) might be systematic because of the overestimation of dynamical correlation in (MR-)MP2 compared to (MR-)CISD; on the other hand, the missing part of the dynamical correlation at the MR-CISD level (i.e. $T_{\mathrm{Sel}}=0$ ) would lower the excitation energy of the ionic $S_{1}$ state again. The latter applies also to the effect of completing the basis set. Thus, error cancelation can be expected. Second, the difference between the $S_{1}$ energies of the two test systems (i.e. the shift due to the counter ion) is already converged within $0.1 \mathrm{eV}$ for $T_{\text {Sel }}=10^{-5}$.

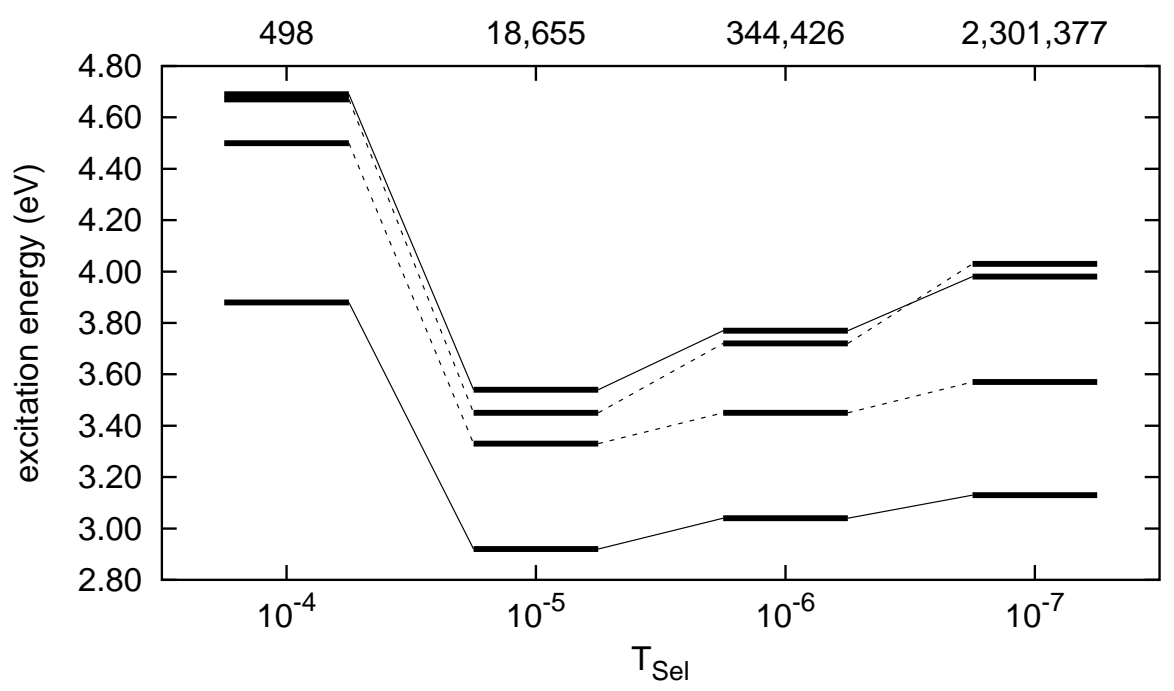

FIG. 5: Dependence of vertical excitation energies $(\mathrm{eV})$ on the selection threshold $T_{\text {Sel }}$ which divides the first-order interacting space (FOIS). Top line: number of configuration state functions in the final variational step. Levels of the system PSB5+Glu133 are connected via dashed lines. 


\section{BASIS SET CONVERGENCY OF SORCI EXCITATION ENERGIES}

Finally we investigated the basis set convergency of excitation energies for several retinal models. For a minimal PSB3 model (see Table ??), fully converged values are not obtained within standard Pople basis sets. Diffuse augmentation functions and (at least) a triple zeta basis is required to achieve convergency within $0.1 \mathrm{eV}$. Surprisingly, the covalent $\mathrm{S}_{2}$ state profits more from basis set extension than the ionic $\mathrm{S}_{1}$ state. For the larger PSB5 model (4-cis- $\gamma$-methylnona-2,4,6,8-tetraeniminium cation), an augmented splitvalence basis appears to be appropriate, though we used the augmented TZVP set in the article. For the full PSB chromophore, basis set dependency is much less critically, since the density of the conjugated $\pi$-system is even more compact than for PSB5. Therefore, augmentation with diffuse functions might not be necessary and we used the $\mathrm{SV}(\mathrm{P})$ set in this work.

TABLE I: Vertical excitation energies (eV) of PSB3 ( $t Z t$-penta-3,5-dieniminium cation) for various basis sets. The dimension of the orbital space and auxiliary basis for the density fit (RI-approximation) are given.

\begin{tabular}{lcccc}
\hline \hline Basis set & $\operatorname{dim}(\mathrm{MO})$ & $\operatorname{dim}($ Aux $)$ & $\mathrm{S}_{1}$ & $\mathrm{~S}_{2}$ \\
\hline $\mathrm{SV}(\mathrm{P})$ & 100 & 432 & 4.38 & 5.64 \\
TZVP & 162 & 432 & 4.21 & 5.43 \\
aug-TZVP & 248 & 600 & 4.07 & 5.23 \\
aug-TZVPP & 466 & 974 & 4.10 & 5.27 \\
aug-cc-pVTZ & 460 & 1004 & 4.06 & 5.24 \\
6-31G* & 100 & 454 & 4.37 & 5.63 \\
$6-311+\mathrm{G}^{*}$ & 156 & 564 & 4.20 & 5.44 \\
$6-311++\mathrm{G}^{* *}$ & 188 & 636 & 4.18 & 5.41 \\
\hline \hline
\end{tabular}

TABLE II: Vertical excitation energies (eV) of PSB5 (4-cis- $\gamma$-methylnona-2,4,6,8-tetraeniminium cation) for various basis sets. The dimension of the orbital space and auxiliary basis for the density fit (RI-approximation) are given.

\begin{tabular}{lcccc}
\hline \hline Basis set & $\operatorname{dim}(\mathrm{MO})$ & $\operatorname{dim}($ Aux $)$ & $\mathrm{S}_{1}$ & $\mathrm{~S}_{2}$ \\
\hline $\mathrm{SV}(\mathrm{P})$ & 182 & 782 & 3.00 & 3.80 \\
aug-SVP & 379 & 1026 & 2.80 & 3.59 \\
TZVP & 293 & 782 & 2.84 & 3.56 \\
aug-TZVP & 448 & 1084 & 2.79 & 3.52 \\
\hline \hline
\end{tabular}

\section{MULLIKEN POPULATION ANALYSIS OF THE RETINAL-GLU133 CHARGE TRANSFER}

Table III shows atomic Mulliken charges for the counter ion (Glu133) in the Rh model of Ref. 3 (see Fig. 6). The charges have been obtained from SORCI calculations with a SV(P) basis. In contrast to the point charge models (3a-MM), considerable amount of charge transfer occurs from the chromophore to the counter ion. However, the Mulliken charges do not significantly change upon excitation. 


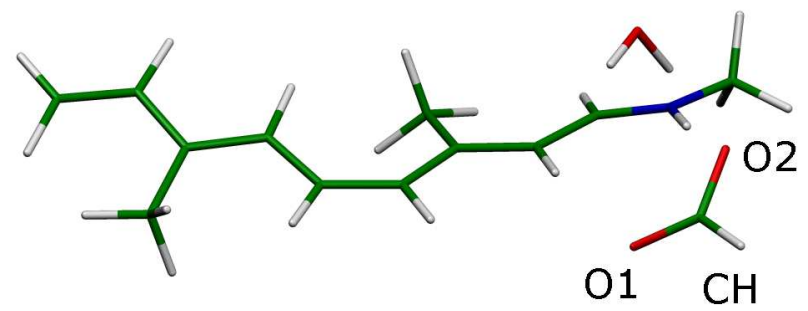

FIG. 6: PSB5 model with Glu133 and one water: "3a-QM" from Ref. 3

TABLE III: Mulliken charges from SORCI/SV(P) for the counter ion in model "3a-QM".

\begin{tabular}{lcc}
\hline \hline & $\mathrm{S}_{0}$ & $\mathrm{~S}_{1}$ \\
$\mathrm{O} 1$ & -0.544 & -0.545 \\
$\mathrm{O} 2$ & -0.720 & -0.719 \\
$\mathrm{C}-\mathrm{H}$ & +0.493 & +0.488 \\
\hline Glu133 & -0.775 & -0.772 \\
\hline \hline
\end{tabular}




\section{CARTESIAN COORDINATES OF STRUCTURES APPEARING IN THE PAPER}

\section{A. All-trans PSB of Retinal}

BLYP/6-31G* optimized $\mathrm{S}_{0}$ minimum.

\begin{tabular}{|c|c|c|c|}
\hline C & 3.671183 & -1.367639 & 0.172512 \\
\hline $\mathrm{C}$ & 3.461123 & 0.177375 & 0.074050 \\
\hline C & 4.541117 & 1.051825 & -0.070647 \\
\hline C & 5.962914 & 0.574209 & -0.287172 \\
\hline C & 6.098164 & -0.920873 & -0.610130 \\
\hline C & 5.187793 & -1.713424 & 0.338190 \\
\hline C & 2.141053 & 0.772924 & 0.102310 \\
\hline C & 0.882559 & 0.185397 & 0.069436 \\
\hline C & -0.355881 & 0.907831 & 0.082520 \\
\hline $\mathrm{C}$ & -0.358641 & 2.425245 & 0.151712 \\
\hline C & 4.437497 & 2.570441 & -0.070178 \\
\hline C & 3.140074 & -2.082019 & -1.110697 \\
\hline C & 2.962811 & -1.945515 & 1.439320 \\
\hline C & -1.546776 & 0.154029 & 0.039623 \\
\hline C & -2.868233 & 0.641424 & 0.052969 \\
\hline C & -3.994003 & -0.196716 & 0.008995 \\
\hline C & -5.351284 & 0.200308 & 0.005006 \\
\hline C & -5.758446 & 1.664853 & 0.044224 \\
\hline C & -6.340954 & -0.820179 & -0.070895 \\
\hline C & -7.713079 & -0.600059 & -0.112856 \\
\hline $\mathrm{N}$ & -8.647180 & -1.572929 & -0.196159 \\
\hline $\mathrm{H}$ & -9.637725 & -1.351998 & -0.232479 \\
\hline $\mathrm{H}$ & -8.120765 & 0.412546 & -0.081317 \\
\hline $\mathrm{H}$ & -6.611813 & 1.828021 & 0.718845 \\
\hline $\mathrm{H}$ & -6.056333 & 2.013627 & -0.959194 \\
\hline $\mathrm{H}$ & -4.946967 & 2.314296 & 0.392818 \\
\hline $\mathrm{H}$ & -3.802107 & -1.274836 & -0.035851 \\
\hline $\mathrm{H}$ & -3.023962 & 1.721507 & 0.095985 \\
\hline $\mathrm{H}$ & -1.431000 & -0.934949 & -0.007087 \\
\hline $\mathrm{H}$ & 0.118777 & 2.773621 & 1.081964 \\
\hline $\mathrm{H}$ & -1.367059 & 2.852761 & 0.118230 \\
\hline $\mathrm{H}$ & 0.210110 & 2.856604 & -0.687581 \\
\hline $\mathrm{H}$ & 0.789828 & -0.899103 & 0.020954 \\
\hline $\mathrm{H}$ & 2.136033 & 1.863391 & 0.112780 \\
\hline $\mathrm{H}$ & 3.656212 & 2.972305 & 0.588776 \\
\hline $\mathrm{H}$ & 4.247673 & 2.949959 & -1.090811 \\
\hline $\mathrm{H}$ & 5.395556 & 3.007349 & 0.249948 \\
\hline $\mathrm{H}$ & 6.534941 & 0.819517 & 0.632305 \\
\hline $\mathrm{H}$ & 6.423590 & 1.202995 & -1.072019 \\
\hline $\mathrm{H}$ & 7.147447 & -1.237871 & -0.502392 \\
\hline $\mathrm{H}$ & 5.821367 & -1.104425 & -1.661997 \\
\hline $\mathrm{H}$ & 5.494175 & -1.502659 & 1.379169 \\
\hline $\mathrm{H}$ & 5.312383 & -2.798892 & 0.191672 \\
\hline
\end{tabular}




$\begin{array}{lrrr}\mathrm{H} & 3.697352 & -1.767631 & -2.006075 \\ \mathrm{H} & 2.077729 & -1.879436 & -1.305217 \\ \mathrm{H} & 3.257668 & -3.172472 & -1.004789 \\ \mathrm{H} & 1.866568 & -1.922352 & 1.386777 \\ \mathrm{H} & 3.266637 & -1.396163 & 2.344378 \\ \mathrm{H} & 3.260493 & -2.997950 & 1.571238 \\ \mathrm{H} & -5.991712 & -1.857762 & -0.111455 \\ \mathrm{H} & -8.388785 & -2.556127 & -0.244205\end{array}$

SCC-DFTB optimized $\mathrm{S}_{0}$ minimum.

$\begin{array}{rrrr}\mathrm{C} & 3.651581 & -1.326897 & 0.200126 \\ \mathrm{C} & 3.441978 & 0.185969 & 0.054422 \\ \mathrm{C} & 4.512325 & 1.042087 & -0.149491 \\ \mathrm{C} & 5.917148 & 0.568871 & -0.309312 \\ \mathrm{C} & 6.034681 & -0.917879 & -0.544697 \\ \mathrm{C} & 5.137823 & -1.649485 & 0.422432 \\ \mathrm{C} & 2.131714 & 0.774030 & 0.118336 \\ \mathrm{C} & 0.901644 & 0.149663 & 0.042306 \\ \mathrm{C} & -0.338996 & 0.849317 & 0.118497 \\ \mathrm{C} & -0.350729 & 2.321972 & 0.338284 \\ \mathrm{C} & 4.351297 & 2.523105 & -0.254967 \\ \mathrm{C} & 3.174346 & -2.050216 & -1.061474 \\ \mathrm{C} & 2.909299 & -1.857749 & 1.429680 \\ \mathrm{C} & -1.518923 & 0.100181 & -0.005891 \\ \mathrm{C} & -2.825215 & 0.608631 & 0.039937 \\ \mathrm{C} & -3.942092 & -0.224246 & -0.082884 \\ \mathrm{C} & -5.285638 & 0.191251 & -0.050488 \\ \mathrm{C} & -5.676611 & 1.620243 & 0.103706 \\ \mathrm{C} & -6.294041 & -0.800194 & -0.169150 \\ \mathrm{C} & -7.654799 & -0.522248 & -0.150397 \\ \mathrm{~N} & -8.585408 & -1.478959 & -0.258990 \\ \mathrm{H} & -9.583666 & -1.250774 & -0.243178 \\ \mathrm{H} & -8.031769 & 0.506067 & -0.044721 \\ \mathrm{H} & -6.264169 & 1.765348 & 1.021236 \\ \mathrm{H} & -6.301974 & 1.945326 & -0.739348 \\ \mathrm{H} & -4.810053 & 2.286119 & 0.152921 \\ \mathrm{H} & -3.758501 & -1.302024 & -0.211404 \\ \mathrm{H} & -2.974803 & 1.689318 & 0.178509 \\ \mathrm{H} & -1.407353 & -0.985286 & -0.153650 \\ \mathrm{H} & 0.139491 & 2.574580 & 1.288949 \\ \mathrm{H} & -1.364820 & 2.732225 & 0.365603 \\ \mathrm{H} & 0.199658 & 2.839269 & -0.460069 \\ \mathrm{H} & 0.831750 & -0.934378 & -0.114020 \\ \mathrm{H} & 2.106282 & 1.872188 & 0.202778 \\ \mathrm{H} & 3.795928 & 2.942687 & 0.595676 \\ \mathrm{H} & 3.811345 & 2.803248 & -1.171769\end{array}$




$\begin{array}{lrrr}\mathrm{H} & 5.329914 & 3.015536 & -0.288654 \\ \mathrm{H} & 6.474927 & 0.852790 & 0.609031 \\ \mathrm{H} & 6.399720 & 1.138199 & -1.127477 \\ \mathrm{H} & 7.083510 & -1.235236 & -0.412376 \\ \mathrm{H} & 5.759240 & -1.158424 & -1.587501 \\ \mathrm{H} & 5.421456 & -1.377252 & 1.456419 \\ \mathrm{H} & 5.284665 & -2.740500 & 0.332681 \\ \mathrm{H} & 3.725507 & -1.705682 & -1.944923 \\ \mathrm{H} & 2.107499 & -1.888489 & -1.253008 \\ \mathrm{H} & 3.335646 & -3.130937 & -0.962139 \\ \mathrm{H} & 1.820187 & -1.806806 & 1.329010 \\ \mathrm{H} & 3.192298 & -1.292805 & 2.326434 \\ \mathrm{H} & 3.174441 & -2.908877 & 1.598143 \\ \mathrm{H} & -5.980444 & -1.847859 & -0.278420 \\ \mathrm{H} & -8.319170 & -2.464642 & -0.359215\end{array}$

B3LYP/6-31G* optimized $S_{0}$ minimum.

$\begin{array}{lrrr}\text { C } & 3.656374 & -1.353818 & 0.171800 \\ \text { C } & 3.439444 & 0.177490 & 0.075087 \\ \text { C } & 4.497814 & 1.052057 & -0.074399 \\ \text { C } & 5.916546 & 0.590123 & -0.286372 \\ \text { C } & 6.058589 & -0.892652 & -0.610408 \\ \text { C } & 5.162980 & -1.685871 & 0.333703 \\ \text { C } & 2.118607 & 0.759147 & 0.107446 \\ \text { C } & 0.878750 & 0.164934 & 0.073115 \\ \mathrm{C} & -0.357693 & 0.879253 & 0.089185 \\ \mathrm{C} & -0.362391 & 2.385792 & 0.164120 \\ \mathrm{C} & 4.386571 & 2.560428 & -0.084699 \\ \mathrm{C} & 3.131836 & -2.065728 & -1.100058 \\ \mathrm{C} & 2.960508 & -1.932015 & 1.429569 \\ \mathrm{C} & -1.534796 & 0.128134 & 0.042572 \\ \mathrm{C} & -2.847719 & 0.615770 & 0.056707 \\ \mathrm{C} & -3.966841 & -0.213410 & 0.008011 \\ \mathrm{C} & -5.311713 & 0.190429 & 0.003947 \\ \mathrm{C} & -5.707621 & 1.647160 & 0.048771 \\ \mathrm{C} & -6.302420 & -0.815847 & -0.075823 \\ \mathrm{C} & -7.661290 & -0.578103 & -0.116114 \\ \mathrm{~N} & -8.602662 & -1.525735 & -0.201027 \\ \mathrm{H} & -9.581424 & -1.288985 & -0.243106 \\ \mathrm{H} & -8.048247 & 0.435543 & -0.080042 \\ \mathrm{H} & -6.542629 & 1.812135 & 0.734383 \\ \mathrm{H} & -6.019490 & 1.990577 & -0.944259 \\ \mathrm{H} & -4.893583 & 2.289985 & 0.380255 \\ \mathrm{H} & -3.781302 & -1.284788 & -0.041031 \\ \mathrm{H} & -2.999175 & 1.688893 & 0.104396 \\ \mathrm{H} & -1.420013 & -0.953180 & -0.008280 \\ \mathrm{H} & 0.119542 & 2.726070 & 1.086476 \\ \mathrm{H} & -1.364484 & 2.810570 & 0.140186 \\ \mathrm{H} & 0.196100 & 2.815885 & -0.673481\end{array}$




$\begin{array}{lrrr}\mathrm{H} & 0.792520 & -0.912562 & 0.020968 \\ \mathrm{H} & 2.105269 & 1.842196 & 0.124095 \\ \mathrm{H} & 3.592904 & 2.963428 & 0.545898 \\ \mathrm{H} & 4.226680 & 2.927255 & -1.107264 \\ \mathrm{H} & 5.328540 & 2.998613 & 0.257436 \\ \mathrm{H} & 6.479179 & 0.833827 & 0.629987 \\ \mathrm{H} & 6.369635 & 1.218091 & -1.065577 \\ \mathrm{H} & 7.103363 & -1.201921 & -0.507898 \\ \mathrm{H} & 5.780108 & -1.075432 & -1.654499 \\ \mathrm{H} & 5.467841 & -1.472267 & 1.367098 \\ \mathrm{H} & 5.294365 & -2.763732 & 0.189349 \\ \mathrm{H} & 3.682062 & -1.749409 & -1.990679 \\ \mathrm{H} & 2.074604 & -1.871191 & -1.291681 \\ \mathrm{H} & 3.255333 & -3.148782 & -0.995584 \\ \mathrm{H} & 1.870951 & -1.915157 & 1.380615 \\ \mathrm{H} & 3.262097 & -1.384110 & 2.327908 \\ \mathrm{H} & 3.262658 & -2.976212 & 1.559759 \\ \mathrm{H} & -5.966350 & -1.849718 & -0.120489 \\ \mathrm{H} & -8.361624 & -2.505129 & -0.254724\end{array}$

BH-LYP/6-31G* optimized $\mathrm{S}_{0}$ minimum.

$\begin{array}{lrrr}\text { C } & 3.633985 & -1.317969 & 0.168581 \\ \text { C } & 3.397449 & 0.179217 & 0.078905 \\ \text { C } & 4.418418 & 1.062835 & -0.066132 \\ \text { C } & 5.823691 & 0.635896 & -0.283190 \\ \text { C } & 5.978299 & -0.822307 & -0.607594 \\ \text { C } & 5.117802 & -1.613063 & 0.334946 \\ \text { C } & 2.077162 & 0.725423 & 0.111620 \\ \text { C } & 0.877367 & 0.100215 & 0.063361 \\ \text { C } & -0.357619 & 0.792174 & 0.082126 \\ \text { C } & -0.367786 & 2.273870 & 0.171897 \\ \text { C } & 4.273338 & 2.545004 & -0.071791 \\ \mathrm{C} & 3.139799 & -2.021487 & -1.084297 \\ \mathrm{C} & 2.954209 & -1.899497 & 1.397847 \\ \mathrm{C} & -1.509260 & 0.043097 & 0.026076 \\ \mathrm{C} & -2.804957 & 0.537306 & 0.047616 \\ \mathrm{C} & -3.915513 & -0.270561 & -0.004786 \\ \mathrm{C} & -5.234562 & 0.161062 & 0.001798 \\ \mathrm{C} & -5.588747 & 1.603599 & 0.056011 \\ \mathrm{C} & -6.239525 & -0.804720 & -0.078374 \\ \mathrm{C} & -7.569759 & -0.502372 & -0.105783 \\ \mathrm{~N} & -8.544169 & -1.383661 & -0.192925 \\ \mathrm{H} & -9.505527 & -1.099133 & -0.213925 \\ \mathrm{H} & -7.892724 & 0.531299 & -0.057759 \\ \mathrm{H} & -6.378422 & 1.788562 & 0.783504 \\ \mathrm{H} & -5.946992 & 1.942274 & -0.918410 \\ \mathrm{H} & -4.748889 & 2.231034 & 0.336025 \\ \mathrm{H} & -3.749429 & -1.341142 & -0.064084 \\ \mathrm{H} & -2.944477 & 1.609409 & 0.106302\end{array}$




$\begin{array}{rrrr}\mathrm{H} & -1.395065 & -1.034309 & -0.036123 \\ \mathrm{H} & 0.117490 & 2.601183 & 1.092680 \\ \mathrm{H} & -1.365928 & 2.699125 & 0.154537 \\ \mathrm{H} & 0.189862 & 2.707109 & -0.660132 \\ \mathrm{H} & 0.815731 & -0.976257 & -0.001854 \\ \mathrm{H} & 2.035114 & 1.806381 & 0.141127 \\ \mathrm{H} & 3.479006 & 2.925977 & 0.565982 \\ \mathrm{H} & 4.099077 & 2.909747 & -1.088163 \\ \mathrm{H} & 5.205815 & 2.999794 & 0.263483 \\ \mathrm{H} & 6.385572 & 0.888658 & 0.626563 \\ \mathrm{H} & 6.254314 & 1.273792 & -1.062104 \\ \mathrm{H} & 7.023849 & -1.120712 & -0.527762 \\ \mathrm{H} & 5.680452 & -1.006492 & -1.642899 \\ \mathrm{H} & 5.412771 & -1.376818 & 1.363549 \\ \mathrm{H} & 5.267073 & -2.687760 & 0.207270 \\ \mathrm{H} & 3.712025 & -1.717472 & -1.961404 \\ \mathrm{H} & 2.092879 & -1.815093 & -1.301870 \\ \mathrm{H} & 3.249559 & -3.101614 & -0.967564 \\ \mathrm{H} & 1.867752 & -1.887375 & 1.351472 \\ \mathrm{H} & 3.253807 & -1.357016 & 2.296122 \\ \mathrm{H} & 3.264746 & -2.939219 & 1.517751 \\ \mathrm{H} & -5.942945 & -1.846289 & -0.133760 \\ \mathrm{H} & -8.350046 & -2.368355 & -0.251107\end{array}$

$\mathrm{HF} / 6-31 \mathrm{G}^{*}$ optimized $\mathrm{S}_{0}$ minimum.

$\begin{array}{lrrr}\mathrm{C} & 3.691557 & -1.334715 & 0.206941 \\ \mathrm{C} & 3.431946 & 0.181112 & 0.051951 \\ \mathrm{C} & 4.426931 & 1.063217 & -0.170055 \\ \mathrm{C} & 5.866855 & 0.657231 & -0.380617 \\ \mathrm{C} & 6.073148 & -0.834637 & -0.583505 \\ \mathrm{C} & 5.200960 & -1.594524 & 0.400955 \\ \mathrm{C} & 2.072535 & 0.735732 & 0.132844 \\ \mathrm{C} & 0.877234 & 0.126670 & 0.071192 \\ \mathrm{C} & -0.396106 & 0.822526 & 0.138375 \\ \mathrm{C} & -0.406005 & 2.319527 & 0.316980 \\ \mathrm{C} & 4.241521 & 2.569707 & -0.257006 \\ \mathrm{C} & 3.208826 & -2.114199 & -1.034705 \\ \mathrm{C} & 3.006037 & -1.893032 & 1.472170 \\ \mathrm{C} & -1.529081 & 0.067582 & 0.032940 \\ \mathrm{C} & -2.862255 & 0.542249 & 0.070999 \\ \mathrm{C} & -3.955819 & -0.273616 & -0.045092 \\ \mathrm{C} & -5.295515 & 0.168838 & -0.013524 \\ \mathrm{C} & -5.599216 & 1.637722 & 0.160663 \\ \mathrm{C} & -6.299078 & -0.796157 & -0.150629 \\ \mathrm{C} & -7.652343 & -0.541156 & -0.146657 \\ \mathrm{~N} & -8.584532 & -1.461398 & -0.277522 \\ \mathrm{H} & -9.548925 & -1.222005 & -0.269940 \\ \mathrm{H} & -8.032176 & 0.456085 & -0.033374 \\ \mathrm{H} & -6.653090 & 1.858943 & 0.202541\end{array}$




$\begin{array}{lrrr}\mathrm{H} & -5.178059 & 2.200555 & -0.664175 \\ \mathrm{H} & -5.154161 & 2.000628 & 1.079216 \\ \mathrm{H} & -3.791108 & -1.328717 & -0.171902 \\ \mathrm{H} & -3.022509 & 1.595707 & 0.196891 \\ \mathrm{H} & -1.408745 & -0.994183 & -0.093110 \\ \mathrm{H} & 0.116731 & 2.587889 & 1.227938 \\ \mathrm{H} & -1.398630 & 2.737022 & 0.372415 \\ \mathrm{H} & 0.107048 & 2.795177 & -0.511609 \\ \mathrm{H} & 0.803978 & -0.934110 & -0.046718 \\ \mathrm{H} & 2.045892 & 1.802056 & 0.227129 \\ \mathrm{H} & 3.666569 & 2.973363 & 0.568166 \\ \mathrm{H} & 3.753633 & 2.858331 & -1.183667 \\ \mathrm{H} & 5.206343 & 3.060104 & -0.238315 \\ \mathrm{H} & 6.433500 & 1.003027 & 0.484698 \\ \mathrm{H} & 6.256365 & 1.218091 & -1.226889 \\ \mathrm{H} & 7.118672 & -1.086842 & -0.438065 \\ \mathrm{H} & 5.827995 & -1.108061 & -1.604851 \\ \mathrm{H} & 5.482709 & -1.301133 & 1.410260 \\ \mathrm{H} & 5.379286 & -2.663150 & 0.328436 \\ \mathrm{H} & 3.392894 & -3.176206 & -0.901866 \\ \mathrm{H} & 3.727336 & -1.794736 & -1.930902 \\ \mathrm{H} & 2.149821 & -1.989694 & -1.220553 \\ \mathrm{H} & 1.927739 & -1.927252 & 1.404545 \\ \mathrm{H} & 3.264970 & -1.300707 & 2.344022 \\ \mathrm{H} & 3.348747 & -2.907936 & 1.648344 \\ \mathrm{H} & -5.990702 & -1.819846 & -0.270533 \\ \mathrm{H} & -8.353186 & -2.423077 & -0.390792\end{array}$

CAS(12,12)/6-31G* optimized $\mathrm{S}_{0}$ minimum. The $\beta$-ionone ring twist angle was constrained to its $\mathrm{HF}$ value during the optimization.

$\begin{array}{lrrr}\text { C } & 3.691391 & -1.337963 & 0.209585 \\ \text { C } & 3.458674 & 0.179653 & 0.033913 \\ \text { C } & 4.490412 & 1.044052 & -0.182418 \\ \text { C } & 5.930366 & 0.606061 & -0.350886 \\ \text { C } & 6.098933 & -0.892933 & -0.545108 \\ \text { C } & 5.192661 & -1.626018 & 0.429886 \\ \text { C } & 2.099108 & 0.763178 & 0.113469 \\ \text { C } & 0.884963 & 0.166801 & 0.043470 \\ \text { C } & -0.399281 & 0.872319 & 0.122995 \\ \text { C } & -0.391923 & 2.370287 & 0.318055 \\ \text { C } & 4.315480 & 2.552375 & -0.287407 \\ \text { C } & 3.214313 & -2.120487 & -1.031699 \\ \text { C } & 2.976928 & -1.874547 & 1.467680 \\ \text { C } & -1.537015 & 0.126831 & 0.015968 \\ \text { C } & -2.900953 & 0.600207 & 0.064847 \\ \text { C } & -3.970742 & -0.232361 & -0.054531 \\ \text { C } & -5.359595 & 0.178388 & -0.013718 \\ \text { C } & -5.679349 & 1.642101 & 0.175931 \\ \text { C } & -6.311175 & -0.801824 & -0.151870\end{array}$




$\begin{array}{lrrr}\mathrm{C} & -7.710391 & -0.583502 & -0.136252 \\ \mathrm{~N} & -8.578979 & -1.537512 & -0.270397 \\ \mathrm{H} & -9.562862 & -1.357125 & -0.255784 \\ \mathrm{H} & -8.126339 & 0.396456 & -0.010746 \\ \mathrm{H} & -6.736747 & 1.859785 & 0.194757 \\ \mathrm{H} & -5.240633 & 2.221826 & -0.627184 \\ \mathrm{H} & -5.256720 & 1.993329 & 1.109503 \\ \mathrm{H} & -3.789408 & -1.284551 & -0.190939 \\ \mathrm{H} & -3.068723 & 1.650666 & 0.200858 \\ \mathrm{H} & -1.423718 & -0.935479 & -0.121167 \\ \mathrm{H} & 0.127222 & 2.630738 & 1.233890 \\ \mathrm{H} & -1.383692 & 2.795202 & 0.374045 \\ \mathrm{H} & 0.126160 & 2.856963 & -0.501157 \\ \mathrm{H} & 0.802644 & -0.892102 & -0.094579 \\ \mathrm{H} & 2.090520 & 1.827782 & 0.228796 \\ \mathrm{H} & 3.833055 & 2.977149 & 0.586865 \\ \mathrm{H} & 3.734291 & 2.836876 & -1.159588 \\ \mathrm{H} & 5.281567 & 3.033959 & -0.377265 \\ \mathrm{H} & 6.490572 & 0.932976 & 0.525225 \\ \mathrm{H} & 6.359388 & 1.145368 & -1.191550 \\ \mathrm{H} & 7.134678 & -1.176286 & -0.385339 \\ \mathrm{H} & 5.858875 & -1.164049 & -1.568429 \\ \mathrm{H} & 5.463805 & -1.328695 & 1.440808 \\ \mathrm{H} & 5.350767 & -2.699031 & 0.371429 \\ \mathrm{H} & 3.385754 & -3.184063 & -0.892291 \\ \mathrm{H} & 3.745110 & -1.811715 & -1.924526 \\ \mathrm{H} & 2.158403 & -1.984971 & -1.228298 \\ \mathrm{H} & 1.899558 & -1.892631 & 1.381554 \\ \mathrm{H} & 3.228677 & -1.277073 & 2.338130 \\ \mathrm{H} & 3.301064 & -2.892744 & 1.661084 \\ \mathrm{H} & -5.987349 & -1.819737 & -0.282955 \\ \mathrm{H} & -8.299902 & -2.491945 & -0.393818\end{array}$

\section{B. 11-cis PSB of Retinal}

B3LYP/6-31G* optimized 6-s-cis $\mathrm{S}_{0}$ minimum. Energy $=-834.1073779486$

$\begin{array}{lrrr}\text { C } & 3.2374575 & -1.4496764 & -0.5266892 \\ \text { C } & 3.1664574 & -0.1546694 & -0.0825916 \\ \text { C } & 4.3647549 & 0.5626221 & 0.5954632 \\ \text { C } & 5.4044530 & -0.4682682 & 1.0947644 \\ \text { C } & 5.7345413 & -1.5338084 & 0.0536970 \\ \text { C } & 4.4676266 & -2.3069095 & -0.3170292 \\ \text { C } & 2.0012889 & 0.6906647 & -0.2762437 \\ \text { C } & 0.6727954 & 0.3524548 & -0.2355612 \\ \text { C } & -0.4117561 & 1.2708531 & -0.4279245 \\ \text { C } & -0.1106477 & 2.7075925 & -0.7758439 \\ \text { C } & 3.8887530 & 1.3655639 & 1.8296976 \\ \text { C } & 5.0267121 & 1.5365234 & -0.4100532\end{array}$




$\begin{array}{lrrr}\mathrm{C} & 2.1367239 & -2.1609941 & -1.2782087 \\ \mathrm{C} & -1.7084978 & 0.7724879 & -0.2740636 \\ \mathrm{C} & -2.8930219 & 1.5135818 & -0.4384283 \\ \mathrm{C} & -4.2387101 & 1.1589991 & -0.3029410 \\ \mathrm{C} & -4.8562054 & -0.0463170 & 0.0944850 \\ \mathrm{C} & -4.0522802 & -1.2571396 & 0.4937369 \\ \mathrm{C} & -6.2694734 & -0.0615635 & 0.1050591 \\ \mathrm{C} & -7.0706466 & -1.1340248 & 0.4538232 \\ \mathrm{~N} & -8.4085959 & -1.1118819 & 0.4450603 \\ \mathrm{H} & 3.2355304 & 2.2042854 & 1.5636008 \\ \mathrm{H} & 3.3466738 & 0.7252643 & 2.5353467 \\ \mathrm{H} & 4.7549963 & 1.7850036 & 2.3545951 \\ \mathrm{H} & 4.3184680 & 2.2854896 & -0.7846080 \\ \mathrm{H} & 5.8447507 & 2.0807272 & 0.0767952 \\ \mathrm{H} & 5.4366711 & 1.0112355 & -1.2787135 \\ \mathrm{H} & 5.0126181 & -0.9610172 & 1.9961931 \\ \mathrm{H} & 6.3099837 & 0.0690544 & 1.4036950 \\ \mathrm{H} & 6.4938120 & -2.2253685 & 0.4363481 \\ \mathrm{H} & 6.1609257 & -1.0714951 & -0.8451243 \\ \mathrm{H} & 4.2271835 & -3.0317075 & 0.4796791 \\ \mathrm{H} & 4.6359952 & -2.9163882 & -1.2143605 \\ \mathrm{H} & 2.5685368 & -2.8296986 & -2.0315410 \\ \mathrm{H} & 1.5478969 & -2.7998846 & -0.6029051 \\ \mathrm{H} & 1.4468007 & -1.4815333 & -1.7825761 \\ \mathrm{H} & 2.2274647 & 1.7473553 & -0.4015106 \\ \mathrm{H} & 0.3946429 & -0.6696453 & 0.0038319 \\ \mathrm{H} & 0.4775064 & 3.1776621 & 0.0216890 \\ \mathrm{H} & 0.4845964 & 2.7652894 & -1.6940026 \\ \mathrm{H} & -1.0036268 & 3.3147861 & -0.9210311 \\ \mathrm{H} & -1.7774268 & -0.2772025 & -0.0148384 \\ \mathrm{H} & -2.7623889 & 2.5508622 & -0.7359426 \\ \mathrm{H} & -4.9325179 & 1.9623199 & -0.5417032 \\ \mathrm{H} & -3.3432272 & -0.9997392 & 1.2877292 \\ \mathrm{H} & -4.6646583 & -2.0824578 & 0.8590273 \\ \mathrm{H} & -3.4724468 & -1.6300273 & -0.3589725 \\ \mathrm{H} & -6.7752377 & 0.8552919 & -0.1941950 \\ \mathrm{H} & -6.6458458 & -2.0840120 & 0.7614491 \\ \mathrm{H} & -8.9237389 & -0.2829839 & 0.1753098 \\ & -8.9507392 & -1.9226533 & 0.7080434\end{array}$

B3LYP/6-31G* $\mathrm{S}_{0}$ minimum with $60^{\circ}$ twisted $\beta$-ionone ring.

$\begin{array}{lrrr}\text { C } & 4.467627 & -2.306909 & -0.317029 \\ \text { C } & 3.237458 & -1.449676 & -0.526689 \\ \text { C } & 3.166457 & -0.154669 & -0.082592 \\ \text { C } & 4.364755 & 0.562622 & 0.595463 \\ \text { C } & 5.404453 & -0.468268 & 1.094764 \\ \text { C } & 5.734541 & -1.533808 & 0.053697 \\ \text { C } & 2.136724 & -2.160994 & -1.278209 \\ \text { C } & 2.001289 & 0.690665 & -0.276244\end{array}$




\begin{tabular}{|c|c|c|c|}
\hline $\mathrm{C}$ & 0.714567 & 0.500316 & 0.158554 \\
\hline C & -0.370845 & 1.408153 & -0.074737 \\
\hline C & -1.602541 & 1.097812 & 0.508524 \\
\hline $\mathrm{C}$ & -2.781215 & 1.855016 & 0.379285 \\
\hline C & -4.062629 & 1.679683 & 0.910509 \\
\hline C & -4.587618 & 0.713537 & 1.795372 \\
\hline $\mathrm{C}$ & -5.958933 & 0.827625 & 2.118096 \\
\hline C & -6.671865 & -0.011195 & 2.955830 \\
\hline $\mathrm{N}$ & -7.973954 & 0.125739 & 3.232394 \\
\hline $\mathrm{C}$ & 3.888753 & 1.365564 & 1.829698 \\
\hline C & 5.026712 & 1.536523 & -0.410053 \\
\hline $\mathrm{C}$ & -0.138236 & 2.636865 & -0.918595 \\
\hline $\mathrm{C}$ & -3.730769 & -0.383297 & 2.373779 \\
\hline $\mathrm{H}$ & 3.235530 & 2.204285 & 1.563601 \\
\hline $\mathrm{H}$ & 3.346674 & 0.725264 & 2.535347 \\
\hline $\mathrm{H}$ & 4.754996 & 1.785004 & 2.354595 \\
\hline $\mathrm{H}$ & 4.318468 & 2.285490 & -0.784608 \\
\hline $\mathrm{H}$ & 5.844750 & 2.080727 & 0.076795 \\
\hline $\mathrm{H}$ & 5.436671 & 1.011236 & -1.278714 \\
\hline $\mathrm{H}$ & 5.012618 & -0.961017 & 1.996193 \\
\hline $\mathrm{H}$ & 6.309983 & 0.069055 & 1.403695 \\
\hline $\mathrm{H}$ & 6.493812 & -2.225368 & 0.436348 \\
\hline $\mathrm{H}$ & 6.160926 & -1.071495 & -0.845124 \\
\hline $\mathrm{H}$ & 4.227184 & -3.031707 & 0.479679 \\
\hline $\mathrm{H}$ & 4.635995 & -2.916388 & -1.214360 \\
\hline $\mathrm{H}$ & 2.568537 & -2.829699 & -2.031541 \\
\hline $\mathrm{H}$ & 1.547897 & -2.799885 & -0.602905 \\
\hline $\mathrm{H}$ & 1.446801 & -1.481534 & -1.782576 \\
\hline $\mathrm{H}$ & 2.207632 & 1.633909 & -0.777398 \\
\hline $\mathrm{H}$ & 0.485034 & -0.370608 & 0.765327 \\
\hline $\mathrm{H}$ & 0.627806 & 3.274342 & -0.460608 \\
\hline $\mathrm{H}$ & 0.225508 & 2.357817 & -1.913825 \\
\hline $\mathrm{H}$ & -1.031071 & 3.247164 & -1.051090 \\
\hline $\mathrm{H}$ & -1.624485 & 0.188255 & 1.096761 \\
\hline $\mathrm{H}$ & -2.708480 & 2.735622 & -0.253781 \\
\hline $\mathrm{H}$ & -4.780906 & 2.429596 & 0.585843 \\
\hline $\mathrm{H}$ & -2.851264 & 0.040790 & 2.869819 \\
\hline $\mathrm{H}$ & -4.252600 & -0.999534 & 3.106950 \\
\hline $\mathrm{H}$ & -3.373127 & -1.049098 & 1.57931 \\
\hline $\mathrm{H}$ & -6.506944 & 1.649974 & 1.66049 \\
\hline $\mathrm{H}$ & -6.201076 & -0.855209 & 3.44935 \\
\hline $\mathrm{H}$ & -8.525478 & 0.874574 & 2.83204 \\
\hline $\mathrm{H}$ & -8.451332 & -0.514242 & 3.8512 \\
\hline
\end{tabular}

B3LYP/6-31G* $\mathrm{S}_{0}$ minimum with $90^{\circ}$ twisted $\beta$-ionone ring.
C $\quad 4.467627$
$-2.306909$
$-0.317029$
C $\quad 3.237458$
$-1.449676$
$-0.526689$
C $\quad 3.166457$
$-0.154669$
$-0.082592$
C $\quad 4.364755$
0.562622
0.595463 


\begin{tabular}{|c|c|c|c|}
\hline C & 5.404453 & -0.468268 & 1.094764 \\
\hline $\mathrm{C}$ & 5.734541 & -1.533808 & 0.053697 \\
\hline C & 2.136724 & -2.160994 & -1.278209 \\
\hline C & 2.001289 & 0.690665 & -0.276244 \\
\hline C & 0.907993 & 0.853313 & 0.535658 \\
\hline C & -0.193073 & 1.729821 & 0.259798 \\
\hline C & -1.189135 & 1.834003 & 1.234778 \\
\hline C & -2.347860 & 2.625927 & 1.137064 \\
\hline C & -3.406598 & 2.839248 & 2.025056 \\
\hline C & -3.640317 & 2.372249 & 3.336285 \\
\hline C & -4.847132 & 2.779506 & 3.948992 \\
\hline C & -5.273684 & 2.434423 & 5.218897 \\
\hline $\mathrm{N}$ & -6.428579 & 2.835523 & 5.762966 \\
\hline C & 3.888753 & 1.365564 & 1.829698 \\
\hline $\mathrm{C}$ & 5.026712 & 1.536523 & -0.410053 \\
\hline C & -0.224170 & 2.489924 & -1.042973 \\
\hline C & -2.652303 & 1.487858 & 4.052853 \\
\hline $\mathrm{H}$ & 3.235530 & 2.204285 & 1.563601 \\
\hline $\mathrm{H}$ & 3.346674 & 0.725264 & 2.535347 \\
\hline $\mathrm{H}$ & 4.754996 & 1.785004 & 2.354595 \\
\hline $\mathrm{H}$ & 4.318468 & 2.285490 & -0.784608 \\
\hline $\mathrm{H}$ & 5.844750 & 2.080727 & 0.076795 \\
\hline $\mathrm{H}$ & 5.436671 & 1.011236 & -1.278714 \\
\hline $\mathrm{H}$ & 5.012618 & -0.961017 & 1.996193 \\
\hline $\mathrm{H}$ & 6.309983 & 0.069055 & 1.403695 \\
\hline $\mathrm{H}$ & 6.493812 & -2.225368 & 0.436348 \\
\hline $\mathrm{H}$ & 6.160926 & -1.071495 & -0.845124 \\
\hline $\mathrm{H}$ & 4.227184 & -3.031707 & 0.479679 \\
\hline $\mathrm{H}$ & 4.635995 & -2.916388 & -1.214360 \\
\hline $\mathrm{H}$ & 2.568537 & -2.829699 & -2.031541 \\
\hline $\mathrm{H}$ & 1.547897 & -2.799885 & -0.602905 \\
\hline $\mathrm{H}$ & 1.446801 & -1.481534 & -1.782576 \\
\hline $\mathrm{H}$ & 2.048642 & 1.327879 & -1.156674 \\
\hline $\mathrm{H}$ & 0.871103 & 0.326275 & 1.484466 \\
\hline $\mathrm{H}$ & 0.647742 & 3.150878 & -1.119509 \\
\hline $\mathrm{H}$ & -0.182815 & 1.799763 & -1.893048 \\
\hline $\mathrm{H}$ & -1.112306 & 3.109752 & -1.162148 \\
\hline $\mathrm{H}$ & -1.034170 & 1.237688 & 2.125953 \\
\hline $\mathrm{H}$ & -2.468268 & 3.171779 & 0.204820 \\
\hline $\mathrm{H}$ & -4.186614 & 3.488686 & 1.633255 \\
\hline $\mathrm{H}$ & -1.661605 & 1.954535 & 4.065779 \\
\hline $\mathrm{H}$ & -2.927890 & 1.280094 & 5.087492 \\
\hline $\mathrm{H}$ & -2.557049 & 0.524438 & 3.537962 \\
\hline $\mathrm{H}$ & -5.505304 & 3.421023 & 3.364835 \\
\hline $\mathrm{H}$ & -4.678168 & 1.797173 & 5.864527 \\
\hline $\mathrm{H}$ & -7.072918 & 3.432862 & 5.259752 \\
\hline $\mathrm{H}$ & -6.696843 & 2.555705 & 6.695793 \\
\hline
\end{tabular}

B3LYP/6-31G* 6-s-trans $\mathrm{S}_{0}$ minimum. Energy $=-834.1088757876$ 


\begin{tabular}{|c|c|c|c|}
\hline $\mathrm{C}$ & -0.1038242 & 0.3135191 & -5.7804306 \\
\hline C & -0.8045008 & 0.1006977 & -4.4619227 \\
\hline C & -0.1256493 & -0.0755197 & -3.2717380 \\
\hline$c$ & 1.4176569 & -0.2084322 & -3.2274714 \\
\hline C & 1.9973018 & -0.3697864 & -4.6577739 \\
\hline C & 1.3904177 & 0.6000943 & -5.6657863 \\
\hline $\mathrm{C}$ & -2.3111325 & 0.1429310 & -4.6085983 \\
\hline $\mathrm{C}$ & -0.9250665 & -0.1015854 & -2.0676284 \\
\hline C & -0.5523938 & -0.0857275 & -0.7427128 \\
\hline $\mathrm{C}$ & -1.4700218 & -0.0872322 & 0.3542952 \\
\hline C & -0.9248877 & -0.0515473 & 1.6438928 \\
\hline $\mathrm{C}$ & -1.6570176 & -0.0543640 & 2.8424082 \\
\hline C & -1.2510387 & -0.0186037 & 4.1819752 \\
\hline C & 0.0268108 & 0.0402402 & 4.7740379 \\
\hline $\mathrm{C}$ & 0.0726724 & 0.0614032 & 6.1878328 \\
\hline C & 1.2135099 & 0.1169808 & 6.9664221 \\
\hline $\mathrm{N}$ & 1.2158336 & 0.1347494 & 8.3055994 \\
\hline C & 1.8400707 & -1.4857478 & -2.4572938 \\
\hline $\mathrm{C}$ & 2.0607627 & 1.0427216 & -2.5762929 \\
\hline $\mathrm{C}$ & -2.9558520 & -0.1295320 & 0.0888283 \\
\hline C & 1.2854673 & 0.0793634 & 3.9456167 \\
\hline $\mathrm{H}$ & 1.6308474 & -1.4465463 & -1.3859562 \\
\hline $\mathrm{H}$ & 1.3362451 & -2.3705185 & -2.8632189 \\
\hline $\mathrm{H}$ & 2.9201095 & -1.6358704 & -2.5725761 \\
\hline $\mathrm{H}$ & 1.6868904 & 1.2371574 & -1.5671783 \\
\hline $\mathrm{H}$ & 3.1469770 & 0.9095999 & -2.5081264 \\
\hline $\mathrm{H}$ & 1.8696793 & 1.9440078 & -3.1680484 \\
\hline $\mathrm{H}$ & 1.8139452 & -1.3967654 & -5.0054281 \\
\hline $\mathrm{H}$ & 3.0862738 & -0.2513889 & -4.6035939 \\
\hline $\mathrm{H}$ & 1.8700059 & 0.4944113 & -6.6453917 \\
\hline $\mathrm{H}$ & 1.5501618 & 1.6387857 & -5.3503947 \\
\hline $\mathrm{H}$ & -0.2704989 & -0.5904377 & -6.3909221 \\
\hline $\mathrm{H}$ & -0.6259747 & 1.1159453 & -6.3216910 \\
\hline $\mathrm{H}$ & -2.6872893 & 1.1667633 & -4.4705689 \\
\hline $\mathrm{H}$ & -2.5890469 & -0.1556590 & -5.6248252 \\
\hline $\mathrm{H}$ & -2.8528432 & -0.5098555 & -3.9199733 \\
\hline $\mathrm{H}$ & -1.9946715 & -0.0937851 & -2.2397140 \\
\hline $\mathrm{H}$ & 0.4960754 & -0.0578922 & -0.4721895 \\
\hline $\mathrm{H}$ & -3.2639016 & 0.7345443 & -0.5116718 \\
\hline $\mathrm{H}$ & -3.2199442 & -1.0307094 & -0.4769205 \\
\hline $\mathrm{H}$ & -3.5572250 & -0.1253845 & 0.9972420 \\
\hline $\mathrm{H}$ & 0.1570525 & -0.0216524 & 1.6901693 \\
\hline $\mathrm{H}$ & -2.7380371 & -0.0928394 & 2.7356807 \\
\hline $\mathrm{H}$ & -2.0755760 & -0.0391665 & 4.8917304 \\
\hline $\mathrm{H}$ & 1.2770226 & 0.9484982 & 3.2778435 \\
\hline $\mathrm{H}$ & 2.1962390 & 0.1360510 & 4.5427502 \\
\hline $\mathrm{H}$ & 1.3593523 & -0.8171549 & 3.3192129 \\
\hline $\mathrm{H}$ & -0.8814179 & 0.0306939 & 6.7122254 \\
\hline $\mathrm{H}$ & 2.2030546 & 0.1502465 & 6.5223117 \\
\hline
\end{tabular}




$\begin{array}{llll}\mathrm{H} & 0.3551333 & 0.1075765 & 8.8377201 \\ \mathrm{H} & 2.0778495 & 0.1761375 & 8.8301924\end{array}$

\section{QM/MM structures}

DFTB/CHARMM QM/MM optimized geometry of all-trans PSB in bR.

\begin{tabular}{|c|c|c|c|}
\hline C & 1.46032 & -2.58719 & 3.41294 \\
\hline $\mathrm{H}$ & 0.68715 & -3.16400 & 3.94187 \\
\hline $\mathrm{H}$ & 2.03632 & -3.29295 & 2.79834 \\
\hline $\mathrm{H}$ & 2.14220 & -2.12967 & 4.14473 \\
\hline C & 0.82421 & -1.53818 & 2.53815 \\
\hline $\mathrm{H}$ & 1.60294 & -1.02381 & 1.94871 \\
\hline $\mathrm{H}$ & 0.37475 & -0.76357 & 3.19040 \\
\hline C & -0.23748 & -2.07690 & 1.60138 \\
\hline $\mathrm{H}$ & -0.91666 & -2.75962 & 2.14438 \\
\hline $\mathrm{H}$ & 0.21709 & -2.66909 & 0.79166 \\
\hline $\mathrm{C}$ & -1.07797 & -0.94685 & 1.02093 \\
\hline $\mathrm{H}$ & -1.48340 & -0.32624 & 1.84358 \\
\hline $\mathrm{H}$ & -1.92766 & -1.36318 & 0.45276 \\
\hline $\mathrm{N}$ & -0.33922 & -0.08925 & 0.12884 \\
\hline $\mathrm{H}$ & -0.21834 & -0.45446 & -0.82597 \\
\hline $\mathrm{C}$ & 0.14579 & 1.09156 & 0.47166 \\
\hline $\mathrm{H}$ & 0.03538 & 1.38884 & 1.52976 \\
\hline C & 0.65295 & 1.97870 & -0.48917 \\
\hline $\mathrm{H}$ & 0.55297 & 1.67727 & -1.54108 \\
\hline C & 1.08435 & 3.28780 & -0.24665 \\
\hline $\mathrm{C}$ & 1.60150 & 3.74717 & 1.06959 \\
\hline $\mathrm{H}$ & 2.66473 & 4.00546 & 0.96347 \\
\hline $\mathrm{H}$ & 1.53092 & 2.98179 & 1.84978 \\
\hline $\mathrm{H}$ & 1.09164 & 4.64997 & 1.42992 \\
\hline C & 0.99722 & 4.18400 & -1.34603 \\
\hline $\mathrm{H}$ & 0.61282 & 3.76317 & -2.28731 \\
\hline C & 1.26001 & 5.53962 & -1.31068 \\
\hline $\mathrm{H}$ & 1.68469 & 6.00489 & -0.40797 \\
\hline C & 0.96044 & 6.33295 & -2.44395 \\
\hline $\mathrm{H}$ & 0.47552 & 5.80663 & -3.28154 \\
\hline C & 1.27790 & 7.66770 & -2.64671 \\
\hline C & 2.01694 & 8.49153 & -1.64837 \\
\hline $\mathrm{H}$ & 3.09207 & 8.49515 & -1.87849 \\
\hline $\mathrm{H}$ & 1.89949 & 8.11295 & -0.62687 \\
\hline $\mathrm{H}$ & 1.68124 & 9.53542 & -1.65538 \\
\hline C & 0.95164 & 8.18852 & -3.94531 \\
\hline $\mathrm{H}$ & 0.35382 & 7.51235 & -4.57414 \\
\hline C & 1.42994 & 9.35780 & -4.47505 \\
\hline $\mathrm{H}$ & 2.01380 & 9.97966 & -3.77867 \\
\hline $\mathrm{C}$ & 1.30457 & 9.88022 & -5.82377 \\
\hline C & 1.64365 & 11.20246 & -6.02515 \\
\hline $\mathrm{C}$ & 2.06040 & 12.12802 & -4.92812 \\
\hline
\end{tabular}




$\begin{array}{rrrr}\mathrm{H} & 2.78310 & 11.69717 & -4.22426 \\ \mathrm{H} & 1.18324 & 12.45364 & -4.34971 \\ \mathrm{H} & 2.52890 & 13.02370 & -5.34670 \\ \mathrm{C} & 1.58115 & 11.87050 & -7.36451 \\ \mathrm{H} & 2.60444 & 11.88456 & -7.79511 \\ \mathrm{H} & 1.31025 & 12.93341 & -7.23921 \\ \mathrm{C} & 0.64395 & 11.18315 & -8.32479 \\ \mathrm{H} & 0.74408 & 11.61721 & -9.33745 \\ \mathrm{H} & -0.40610 & 11.33775 & -8.02375 \\ \mathrm{C} & 0.98597 & 9.71914 & -8.35063 \\ \mathrm{H} & 2.04503 & 9.61533 & -8.65763 \\ \mathrm{H} & 0.39085 & 9.20096 & -9.12190 \\ \mathrm{C} & 0.77599 & 9.01947 & -6.99288 \\ \mathrm{C} & -0.73122 & 8.78045 & -6.80114 \\ \mathrm{H} & -1.24561 & 9.70791 & -6.52210 \\ \mathrm{H} & -0.94594 & 8.03703 & -6.02397 \\ \mathrm{H} & -1.18587 & 8.41334 & -7.73011 \\ \mathrm{C} & 1.52125 & 7.68335 & -7.12608 \\ \mathrm{H} & 1.35594 & 6.99088 & -6.29424 \\ \mathrm{H} & 2.60261 & 7.84902 & -7.21406 \\ \mathrm{H} & 1.19038 & 7.16234 & -8.03306\end{array}$

* Corresponding author. Email address: m.elstner@dkfz-heidelberg.de

1 Ferre, N.; Olivucci, M. J. Am. Chem. Soc. 2003, 125, 6868-6869.

2 Neese, F. J. Chem. Phys. 2003, 119, 9428.

3 Schreiber, M.; Buss, V.; Sugihara, M. J. Chem. Phys. 2003, 119, 12045-12048. 